\title{
Stereo Based Structure Recovery of Underwater Scenes from Automatically Restored Images
}

\author{
Erickson Nascimento, Mario Campos, Wagner Barros \\ Universidade Federal de Minas Gerais \\ Departamento de Ciência da Computação \\ Belo Horizonte, Brazil \\ \{erickson,mario,wbarros\}@dcc.ufmg.br
}

\begin{abstract}
In this paper we present a fully automatic methodology for underwater image restoration which is based on classical physical models of light propagation in participating media. The technique uses pairs of images acquired from distinct viewpoints under the same environmental conditions. At the kernel of the method is an iterative algorithm that is based on a contrast metric that automatically estimates all parameters of the model with good accuracy at a significantly low computational cost. We then present an algorithm that uses the model with the estimated parameters to improve the quality of images of underwater scenes taken under natural illumination, (i.e. without any special light source). First we show the quality of parameters estimated by our approach by comparing against the same parameters obtained manually like in other works in the literature. Once better estimated parameters greatly influence the quality of restored images, we performed experiments with images taken from both synthesized and real scenes to verify the performance of the proposed method. Two main aspects were considered: image quality and quality of disparity maps produced by a standard stereo algorithm. Image quality was assessed by a quantitative measure of contrast, which is typically used in related literature. We also compare the results obtained by our methodology with those obtained with classic image enhancement tools. The results obtained with our methodology demonstrate improvement both in scene contrast of recovered underwater images and in the accuracy of the disparity maps under different water turbidity levels.
\end{abstract}

Keywords-underwater; physics based vision; stereo; image restoration; visibility; scattering; attenuation

\section{INTRODUCTION}

Computer Vision algorithms have been designed based on assumptions about the environment, one of them being that the medium between the camera and the scene is immaterial. Nonetheless, in several cases this assumption does not hold, such as foggy and rainy days and underwater environments. Visibility in such cases may be strongly reduced to the point that useful images may not be obtained [1] at all.

There is an ever growing volume of underwater imagery being produced in research and actual applications in oceanic engineering and mapping, water fauna identification and assessment [2], [3], [4], autonomous underwater robotics [5], [6], [7], and underwater archaeology [8] to name a few.
In this work we focus on imagery acquired in underwater environments where the interaction of light with the medium produces side effects that are not negligible, such as attenuation and scattering, which are commonly present in underwater scenes. Furthermore, we are interested in automatically reducing, and if possible, canceling the impact of these effects on underwater images.

Several methodologies that deal with underwater images have been proposed in the literature. Broadly speaking, such methodologies may bundled into two main groups: image based and model based approaches. Image processing techniques are typically used in the first case [9], [10], [11], [12]. Since they do not assume any model for spatially varying dependencies and other environmental characteristics, these methods are limited in their ability to recover visibility [13]. In model based approaches, however, light propagation and interaction with the medium are taken into account. There are some works where active illumination hardware is used [14], [15], images of the same scene taken with the medium in different conditions [16], dense stereo algorithm [17] or different states of a polarizing filter [15], [18]. Even though a light propagation model is used, those methods either require expensive equipments such as those used in [14], [16], [15], or are very time consuming like the work reported in [16] and [17], where two images of the same scene with significantly distinct properties may take several days to be acquired and the computation of model's parameters uses complex and expensive optimization algorithms respectively. Furthermore, with except of [17], most of these methodologies are not amenable to realtime applications, such as underwater live species identification or autonomous underwater robot navigation and guidance [5], [6], [7].

This paper presents a methodology, which like other works such as [13] and [17], is based on a physical model that describes light propagation in water proposed in [19], that is used to estimate this model's parameters in order to minimize the effects on image formation. One of the key issues is the estimation of the model parameters, which frequently is performed by a manually ad-hoc procedure. Our technique builds upon the approach described in [17], and as in that work, we use pairs of images acquired 
from distinct viewpoints under the same environmental conditions. However, we introduce a novel procedure that automatically estimates all model parameters with better accuracy and with a low computational cost. The is used to both recover. Based on this calibrated model, we design an algorithm which improves the quality of images of underwater scenes taken under natural illumination, i.e. without any special lighting, thus enhancing scene visibility which enables the accomplishment of tasks such as the reconstruction underwater scenes.

The quality of the results are demonstrated by comparing the model's parameters obtained automatically against those fine-tuned manually. A contrast metric [13], computed on images restored by our methodology and on classic image enhancement tools, quantifies the improvement introduced by our methodology.

\section{IMAGE FORMATION MODEL}

Light rays traversing a liquid medium will interact with it generating different artifacts which will affect characteristics such as intensity and color at the sensor. There are two main effects caused thereof, namely, absorption and scattering as illustrated in Fig. 1. The physical process in which light rays interacting with particles in suspension in the medium is converted into other forms of energy is called absorption. As a result, the intensity of a point in the image will decay as the distance between the scene and the camera increases. Scattering is caused by the change in direction of a ray of light after colliding with a particle in suspension. For small angular changes in direction the effect is called forward scattering), whereas for larger angles up to 180 degrees, which effectively causes the ray to bounce back at the camera, it is called backscattering. As a result of the scattering effect, objects located farther away from the camera appear to be brighter. Therefore, a suitable model for light propagation underwater should incorporate, at least, these two effects.

Our approach assumes that as far as image formation is concerned, both effects are additive, and as such, they can be combined into a simple sum. Thus, the intensity $I$ of a pixel at position $(x, y)$ may be written as:

$$
I(x, y)=L(x, y)+B(x, y)
$$

where $L(x, y)$ is the component of the light ray attenuated by absorption and $B(x, y)$ is the contribution of the backscattering effect. Our model is an approximation of the Equation of Radiative Transfer [20] and it is inspired in the works described in [13], [17] and [21].

\section{A. Attenuated light}

Consider the radiance of a narrow light beam, like a collimated beam, emanating from a source and traveling through a medium. The decrease in radiance $\Delta L$ is due to

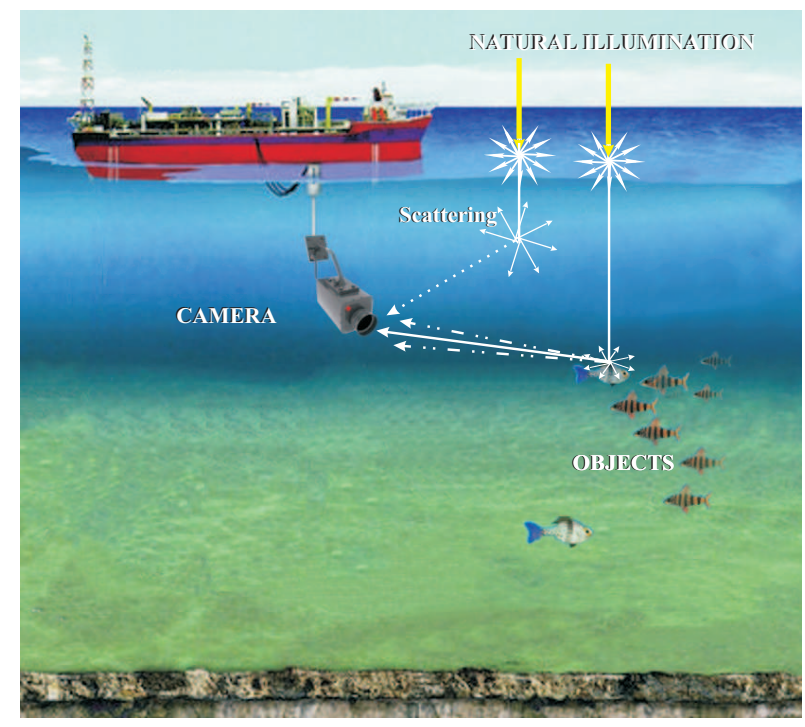

Figure 1. Components of light propagating in a underwater environment: direct component (-), light ray which is reflected by an object's surface $(\cdots)$ and the backscattered component $(-\cdots-)$, the light scattered by the medium.

the interaction of the light ray with the medium. For an infinitesimal distance $\Delta x$ we have that

$$
\frac{\Delta L(x)}{L(x)}=-c \Delta x,
$$

where $c$ is the attenuation coefficient, given in $m^{-1}$ units. The attenuation coefficient is a function of the wavelength $\lambda$ and is given by the sum of the absorption and the total scattering coefficients. The latter represents the capability an infinitesimal volume has to scatter flux in all directions.

By integrating both sides of Eq. 2 between the limits $x=$ 0 and $x=z$ we obtain:

$$
L(z)=L_{0} e^{-c z},
$$

which is known as Bouguer's exponential law of attenuation [19], where $L_{0}$ is the object's radiance without attenuation and $z$ is the distance traveled by the collimated beam.

\section{B. Scattered light}

In order to model the scattering effect, consider a cone with its vertex at the observer's position and truncated by the surface of a physical object at distance $d$ (Fig. 2). The irradiance due to scattering in the direction of the observer, produced by an infinitesimal volume at distance $z$, may be described as [21]:

$$
d B(z)=\frac{d I(z) e^{c} z}{d \omega z^{2}}
$$

where $d \omega$ is the cone's solid angle, $c$ is the total scattering coefficient and $d I(z)$ is the intensity of the infinitesimal. 


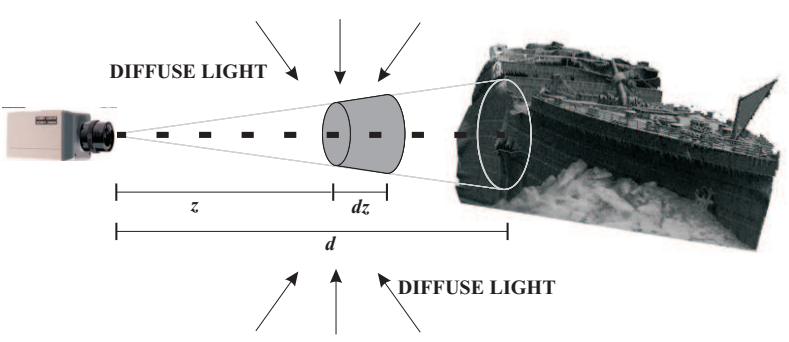

Figure 2. The cone between the observer and an object immersed in water. The backscattering light increase the brightness of objects in pathlength acting like a source of light.

Here $d I(z)=d \omega z^{2} d z \beta c$, where $\beta$ is called Volume Scattering Function. This function describes the nature of the illumination and the characteristics of the scattering.

The total radiance along the path between the observer and the object is calculated by integrating both side of Eq. 4 within the limits $x=[0, d]$, which results in:

$$
B(d)=\beta\left(1-e^{-c d}\right) .
$$

For infinite distances $(d=\infty)$, such as objects in the horizon, the radiance received by the camera's sensor is $B(\infty)=\beta$. Re-writing Eq. 5 we have:

$$
B(d)=B_{\infty}\left(1-e^{-c d}\right) .
$$

Substituting $B(d)$ and $L(d)$ in Eq. 1 and using $z$ as depth we obtain the model:

$$
I(x, y, z)=L_{0} e^{-c z}+B_{\infty}\left(1-e^{-c z}\right) .
$$

where $I(x, y, z)$ is the intensity of pixel $(x, y)$ at depth $z$.

\section{VISIBILITY RECOVERY}

The main goal of this section is to show that the model described by Eq. 7 can be successfully used to recover the visibility of underwater scenes and improve the quality of disparity maps. The model requires that the depth of each point in the scene and the parameters $c$ and $B_{\infty}$ be known. As depicted in Figure 3, our methodology consists of three main steps: i) compute the scene's depth; ii) estimate the model's parameters, and iii) recover the visibility (or estimate the object's radiation for a transparent medium). These steps are executed iteratively while the attenuation coefficient, estimated in the second step, is greater than zero.

\section{A. Computing scene depths}

Like in [17], the depths maps are obtained from a stereo pair obtained from two geometrically calibrated cameras. The disparity map was obtained using Kolmogorov and Zabih's algorithm [22] due to its ability to handle occlusions. Using the disparity maps and the geometrical parameters, we estimate the depths of each pixel of the images of left

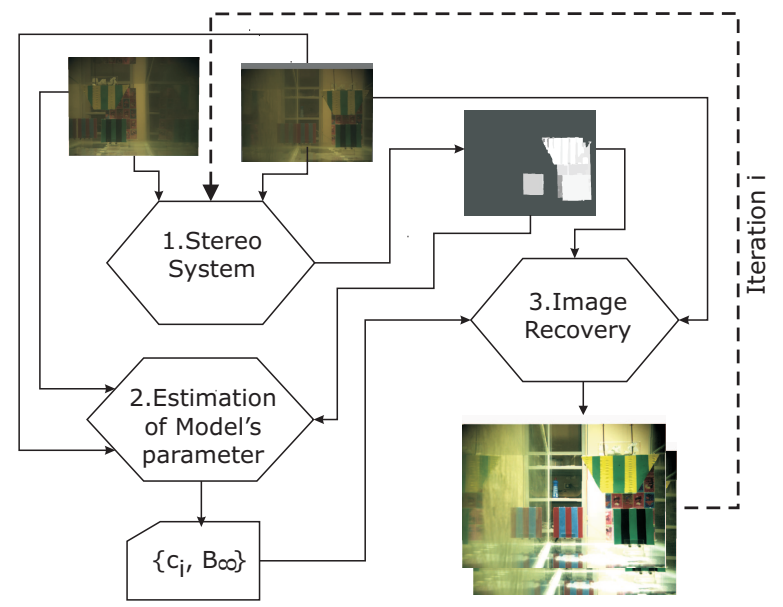

Figure 3. Image restoration process. Two images taken from an underwater scene are used for depth map estimation, after which the computed disparity maps are used to estimate the model's parameters. Based on the depth maps and the model's parameters the image is finally restored.

and right cameras. In experiments performed, we used a baseline of $33 \mathrm{~cm}$ and the average distance from the cameras reference to scene was $140 \mathrm{~cm}$.

The correspondence between pixels, one of the key problems with stereo algorithms, is severely aggravated in underwater environments. The methodology handle this in each iteration. After $c_{i}$ is estimated in iteration $i$, another correspondence process is performed to find a new disparity map. As some of underwater effects were removed in i-1th iteration, the difference between corresponding pixels, due to attenuation and scattering effects, will be minimized.

\section{B. Estimating the model's parameters}

Given a pair of rectified stereo images of the same scene was acquired, for each pixel at location $(x, y)$ the intensities of each pixel for the left and right cameras are $I_{l}$ and $I_{r}$, respectively. Since absorption and scattering effects are a function of distance, the intensity $I$ of corresponding pixels in both images of the same scene point tends to be different because the distances $z_{r}$ and $z_{l}$ of a scene point to the right and left cameras are different. Assuming the water properties to be the same for both images (they are captured synchronously) and assuming that $B_{\infty}$ is the same regardless of position, for each scene point we can write $I_{l}$ and $I_{r}$ as:

$$
\begin{aligned}
& I_{l}(x, y, z)=L_{0} e^{-c z_{l}}+B_{\infty}\left(1-e^{-c z_{l}}\right) \\
& I_{r}(x, y, z)=L_{0} e^{-c z_{r}}+B_{\infty}\left(1-e^{-c z_{r}}\right) .
\end{aligned}
$$

Rewriting Eq. 7 as:

$$
\ln \left(I(x, y, z)-B_{\infty}\right)=-c z+\ln \left(L_{0}-B_{\infty}\right)
$$

and solving the system of Equations 8 and 9, we find that: 


$$
\ln \left(I_{r}-B_{\infty}\right)-\ln \left(I_{l}-B_{\infty}\right)=-c\left(z_{l}-z_{r}\right) .
$$

Hence $c$, the attenuation coefficient, may be estimated by fitting a line to the points given by Eq. $11 . B_{\infty}$ can be extracted from the observed intensity of points in the scene's horizon, like in [16], [18], [21].

\section{Recovering the visibility}

Recovering visibility is a straightforward process. The radiance that an object would have if it was immersed in a transparent medium is given by:

$$
L_{0}=\frac{I(x, y, z)-B_{\infty}\left(1-e^{-c z}\right)}{e^{-c z}} .
$$

\section{EXPERIMENTS}

Several experiments were carried out, both in simulation and in a real environments, in order to evaluate the proposed methodology.

As mentioned before, our approach has a module to compute pixel depth using a stereo image pair. Alos, as we mentioned, the disparity map is obtained by running Kolmogorov and Zabih's algorithm [22], and we fit the data to Eq. 11 using the linear least squares technique.

\section{A. Generating Synthetic Scenes}

Synthetic scenes with objects at different locations were generated with [23], and the real depth information was obtained for each pixel. For each scene, two images were synthesized from two different viewing angles, thus simulating a stereo pair. One pair of images from the synthetic scene were obtained for each value of the absorption coefficient $c$, simulating different turbidity levels. A zero mean gaussian noise was added to the pixel intensity ( $\sigma=3$ pixels) and depth information ( $\sigma=2$ meters).

The quality of disparity maps computed by our methodology was measured using RMS (root-mean-squared) error and the percentage of bad matching pixels based on a known ground truth scene. As described in [24], the RMS error between the computed disparity map $d_{c}(x, y)$ and ground truth map $d_{g t}(x, y)$ is given by:

$$
R=\left(\frac{1}{N} \sum_{x, y}\left|d_{c}(x, y)-d_{g t}(x, y)\right|^{2}\right)^{\frac{1}{2}}
$$

where $\mathrm{N}$ is the number of pixels.

Table I shows the results obtained with the simulation. We used both low $\left(c=0.13 \mathrm{~m}^{-1}\right)$, medium $\left(c=0.21 \mathrm{~m}^{-1}\right)$ and high turbidity $\left(c=0.39 \mathrm{~m}^{-1}\right)$ conditions. The stereo algorithm we called regular stereo is the one described in [22], and it was chosen because due to its ability in dealing with occlusions. As it can be seen, our methodology produced disparity maps with small RMS error.
Table I

SiMULATION RESULTS. COMPARING THE RMS ERROR BETWEEN THE DISPARITY MAPS PRODUCED BY A REGULAR STEREO $\left(R M S_{r}\right)$ AND USING OUR UNDERWATER METHODOLOGY $\left(R M S_{u w}\right)$.

\begin{tabular}{c|c|c}
\hline$c\left(\mathrm{~m}^{-1}\right)$ & $R M S_{r}($ pixels $)$ & $R M S_{u w}($ pixels $)$ \\
\hline \hline 0.13 & 157.56 & 154.65 \\
0.21 & 177.53 & 159.23 \\
0.39 & 174.55 & 168.31 \\
\hline
\end{tabular}

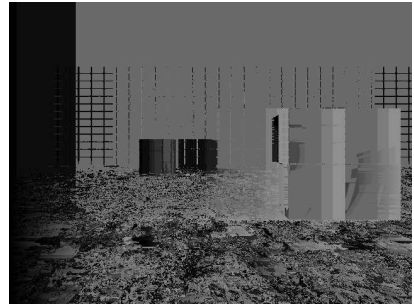

(a)

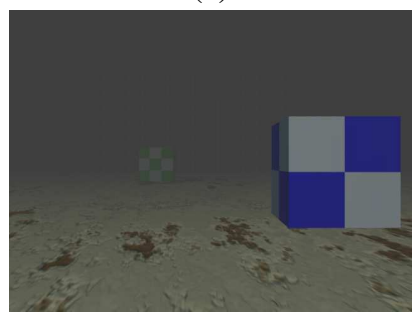

(c)

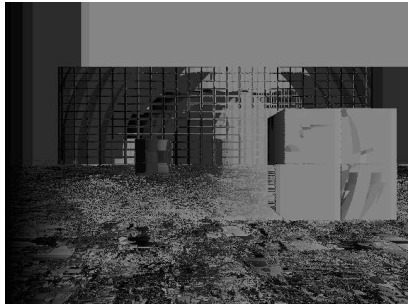

(b)

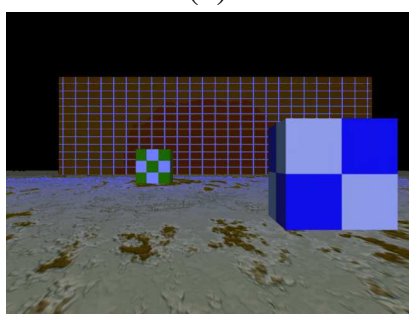

(d)
Figure 4. (a) Disparity map using a regular stereo; (b) using our underwater approach; (c) underwater image simulation of high turbidity $\left(c=0.39 m^{-1}\right)$ and the restored image (d). Observe how the brick wall becomes visible after the recovering process and the disparity information of the scene is more accurate with the use of our underwater methodology. The disparity values of the brick wall and the background are pratically the same for the regular stereo in (a), and are very distinct in the map produced by our underwater stereo technique (b).

Fig. 4 depicts underwater images that were restored using our methodology, and the disparities maps computed using a regular stereo and the our underwater methodology. It can be readily seen that even objects farther away in the scene, such as the brick wall, in a high turbidity condition, becomes visible after restoration and presents better disparity values.

\section{B. Real Underwater Scene}

Several experiments were performed with real imagery obtained from the experimental set-up depicted in Fig. 5. Two geometrically calibrated Dragon Fly cameras [25] ( $\mathrm{f}=8$ $\mathrm{mm}$, resolution of $640 \times 480$ at 30 FPS), with a a baseline of $33 \mathrm{~cm}$ were positioned fronto-parallel facing the smaller side of a glass fish tank measuring $168 \mathrm{~cm} \times 47 \mathrm{~cm} \mathrm{x}$ $45 \mathrm{~cm}$, which accommodates 320 liters of water. Two sets of experiments were performed using multiple objects with simple shapes. Besides the images acquired in clear water, different amounts of green tea were dissolved in the tank water to simulate two levels of turbidity: low ( $70 \mathrm{~g}$ of green tea) and high (110g of green tea). All the experiments were performed under controlled lighting. The only illumination 


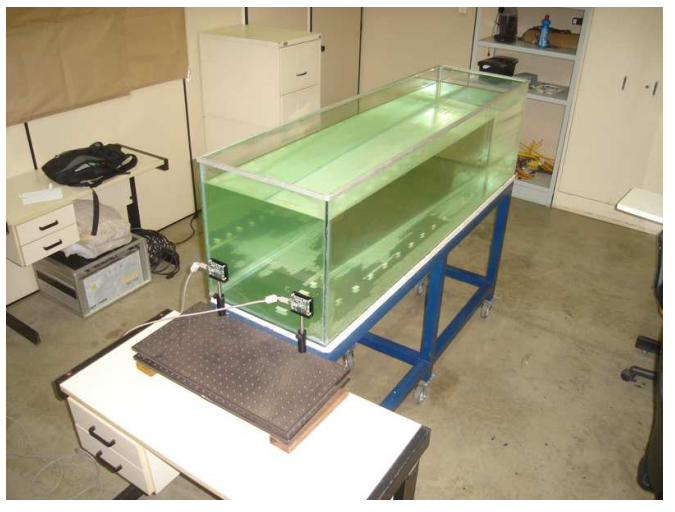

Figure 5. Experimental setup. The fish tank has dimensions $168 \mathrm{~cm} \mathrm{x}$ $47 \mathrm{~cm} \mathrm{x} 45 \mathrm{~cm}$ and is filled with 320 liters of water. A pair of calibrated Dragon Fly cameras with a baseline of $33 \mathrm{~cm}$ is positioned front-parallel facing the smaller side of the fish tank.

source was composed of four pairs of fluorescent lamps disposed 2 meters above the fish tank.

The value for the attenuation coefficient was estimated using both manual and automatic approaches. The manual approach consists in computing the average intensity of the pixels corresponding to the target's black patch (like in [15] and [17]) in an image acquired with the known object positioned at a known distance $z$ from the cameras and fitting a line to the following equation:

$$
c z=-\ln \left(\frac{B_{\infty}-I(z)}{B_{\infty}}\right),
$$

note that it is necessary to know original radiance and each position that the object was placed.

Results using the value of $c$ estimated manually and our automatic methodology can be qualitatively compared by observing Fig.7. It is important to note that the values estimated by the manual approach is not considered to be the ground truth. We chose this comparison method because it is a standard procedure and because it is easy to use with manual approaches to obtain the attenuation coefficient $c$.

Similarly to the criteria used in [18], we evaluated the recovered images by performing a contrast analysis. This metric can be considered as a reasonable performance index for restored underwater images and its use is justified because stereoscopic acuity and the determination of the MTF (Modulation Transfer Function) [26] are related to contrast measurement.

The procedure to compute the contrast was based on equations described in [18]. For an image $I$ with $N$ pixels, the contrast $C(I)$ can be estimated by

$$
C(I)=\frac{\sqrt{\frac{1}{N} \sum_{v=1}^{N} \sum_{\chi=r, g, b}\left(I_{v}^{\chi}-\bar{I} \chi\right)^{2}}}{\sum_{\chi=r, g, b} \bar{I}^{\chi}},
$$

where $\chi$ is the index of the chromatic band (red, green and blue) and

$$
\bar{I}^{\chi}=\frac{1}{N} \sum_{v=1}^{N} I_{v}^{\chi} .
$$

Tables II and III show the improvement obtained with the restoration using the value of $c$ estimated with our methodology, which is completely automatic, and with the corresponding value which was obtained manually. The position of each object in the scene is known a priori. This enabled us to compute the contrast of the surface of each object, which corresponds to a fixed distance to the camera. The distances and their respective contrast values are presented in the tables below.

As it can be seen from the data, there was an improvement in image contrast for all experimental data sets. Observe that for objects located farther away from the camera there was an increase in the contrast similar to nearby objects. For images recovered using histogram equalization one may observe that there is a decrease in the contrast when objects are distant from the camera. This behavior is clearly seen in experiments under high turbidity conditions (Table III). For the farther away object in the higher turbidity trial, our methodology obtained a increase of $7.64 \%$ in the contrast value while the histogram equalization technique was only $3.61 \%$.

Table II

THE CONTRAST OF THE RAW IMAGE $C(I)$ FOR MEDIUM TURBIDITY (70G OF GREEN TEA DISSOLVED IN 320 LITERS OF WATER), AND THE RECOVERED IMAGES WITH HISTOGRAM EQUALIZATION $C\left(I^{\text {hist }}\right)$, AND BY OUR METHODOLOGY $C(\hat{I})$.

\begin{tabular}{c|c|c|c}
\hline Distance $(\mathrm{m})$ & $C(I)(\%)$ & $C\left(I^{\text {hist }}\right)(\%)$ & $C(\hat{I})(\%)$ \\
\hline \hline 1.10 & 17.47 & 24.25 & 25.69 \\
1.20 & 14.00 & 59.99 & 29.15 \\
1.40 & 14.28 & 45.86 & 27.42 \\
1.60 & 7.42 & 18.92 & 18.33 \\
\hline
\end{tabular}

The images in Fig.7 depicts the result of the disparity map estimation using the methodology that computes $c$ by a manual approach (Figs. 7 (b) and (e)) and by the automatic method described in this paper (Figs. 7 (c) and (f)). It can be seen that the disparity maps for both techniques are quite similar, and there is a significant enhancement in the map quality when compared to the one produced by a regular stereo algorithm (Figs. 7 (a) and (d)). It can be seen that

Table III

THE CONTRAST OF THE RAW IMAGE $C(I)$ FOR HIGH TURBIDITY $(110 \mathrm{G}$ OF GREEN TEA DISSOLVED IN 320 LITERS OF WATER), AND THE RECOVERED IMAGES WITH HISTOGRAM EQUALIZATION $C\left(I^{\text {hist }}\right)$, AND BY OUR METHODOLOGY $C(\hat{I})$.

\begin{tabular}{c|c|c|c}
\hline Distance $(\mathrm{m})$ & $C(I)(\%)$ & $C\left(I^{\text {hist }}\right)(\%)$ & $C(\hat{I})(\%)$ \\
\hline \hline 1.10 & 14.94 & 24.66 & 28.67 \\
1.20 & 8.42 & 42.01 & 23.29 \\
1.40 & 11.48 & 39.40 & 24.68 \\
1.60 & 5.29 & 8.90 & 12.93 \\
\hline
\end{tabular}




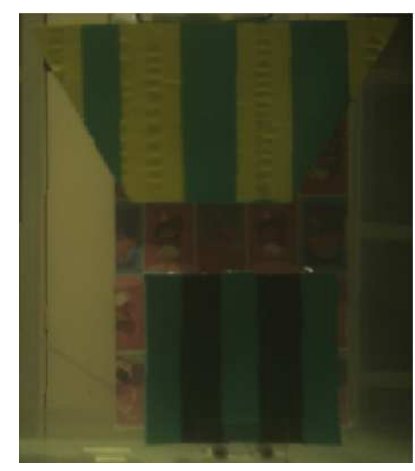

(a)

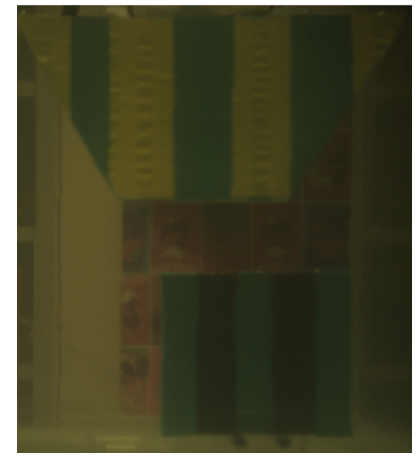

(c)

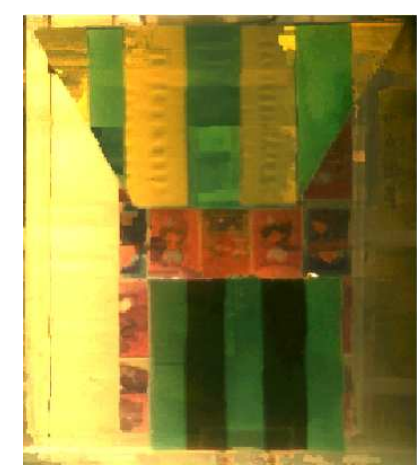

(b)

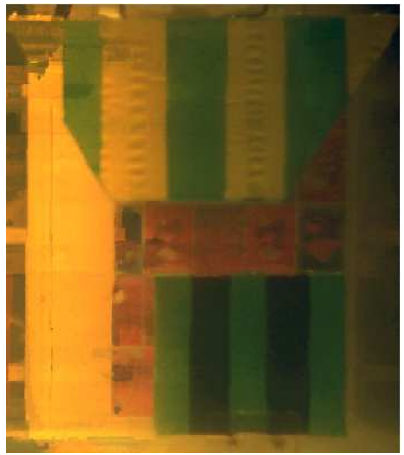

(d)

Figure 6. Results of our underwater image recovering algorithm for a real scene immersed in (a,b) $70 \mathrm{~g}$ and (c,d) $110 \mathrm{~g}$ dissolved tea in water. Figures (a) and (c) are the acquired images of left camera and (b) and (d) are their respectively recovered versions.

the disparities of the object with green and black strips is incorrectly computed by the regular stereo algorithm. However, the values estimated with our underwater approach (with model parameters computed automatically) are clearly more accurate.

From the restoration results shown in Fig. 6 we are able to observe a number of artifacts and distortions that can be directly attributed to the depth map computed from the stereo pairs obtained from the scene. This shows that our methodology is quite robust to noise, artifacts and inaccuracies in the depth maps estimated from stereo pairs. If depth maps are not sufficiently accurate, it is still possible to achieve good quality restoration. In such cases, if the relative distances between the scene background and the camera is small, as we happen to have in our experiments, we can consider the mean value of the distances to restore the whole image instead of using the depth map. Thus, we are able to retrieve the real color (the color of the scene as imaged without water) of a large area of the image and we can also avoid problems related to color saturation in the image. These features of our methodology come to par with the major challenges for conventional methods for image enhancement using digital image processing techniques alone.

\section{Conclusions}

We have presented a methodology that addresses the problem caused by images degradation effects typically present in underwater scenes. Our method builds upon a physical model that explains the light propagation in liquid medium. The technique does not require any calibration of the environmental parameters and can be used with other Computer Vision algorithms to recover the visibility of underwater scenes. The methodology was used with a stereo algorithm, however it can be readily adapted to work with other algorithms which provide depth information, such as depth from motion. All parameters in the model are estimated automatically. We performed experiments with simulations and real scenes to verify the quality of the results obtained with the proposed method. As it can be can be seen in Figs. 7 and 6, as well as in simulation results, our methodology is able to greatly improve the quality of the disparity map. The methodology seems to have good potencial to restore underwater images of scenes under a wide spectrum of turbidity levels. For environments were artificial light sources are used, as is deep-water exploration, the methodology may fail. The second limitation is the method's dependence on depth estimation. If an estimation of the scene is not available, the method may produce large errors and the model parameters may not be correctly 


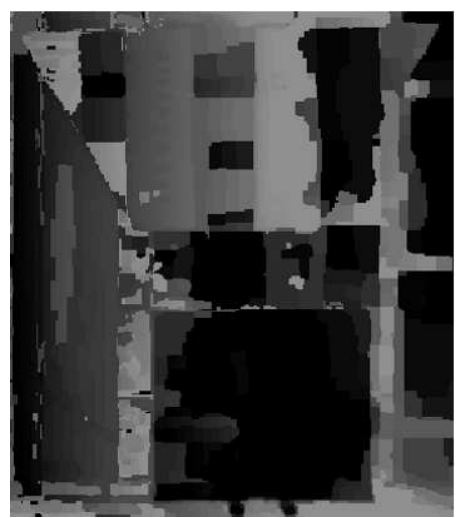

(a)

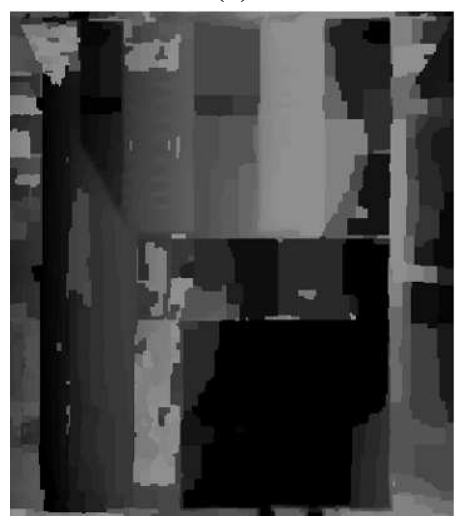

(d)

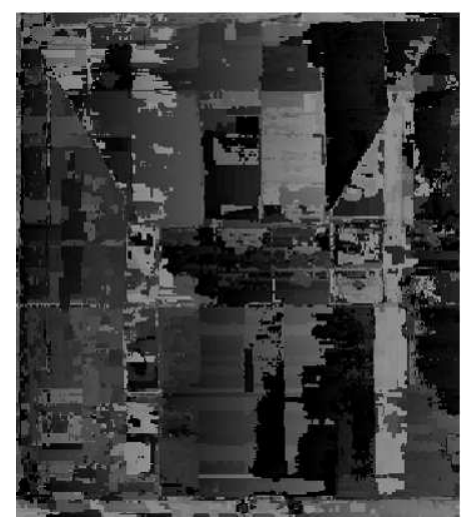

(b)

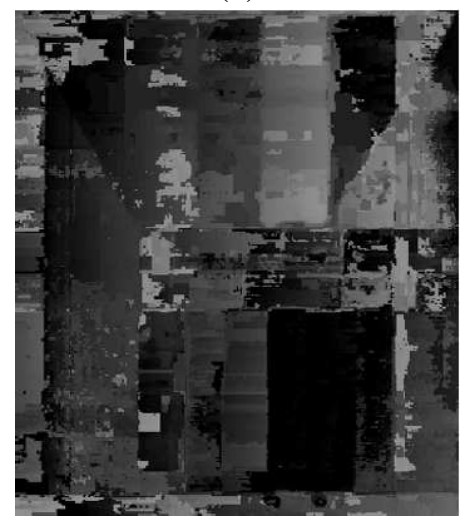

(e)

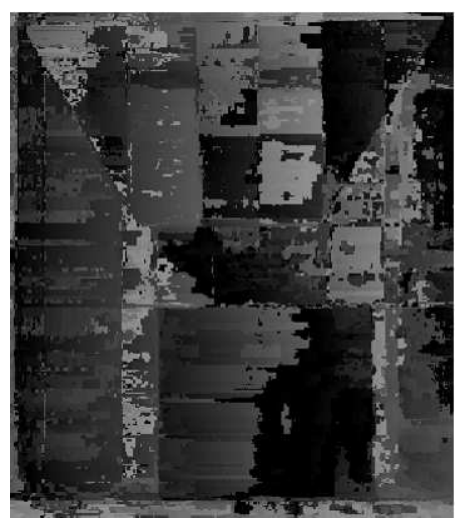

(c)

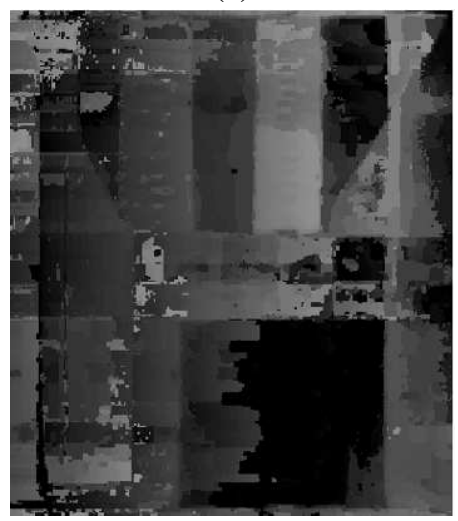

(f)

Figure 7. Results for disparity map estimation for a real underwater scene with $70 \mathrm{~g}$ (a,b,c), and with $110 \mathrm{~g}$ (d,e,f) of tea dissolved in water. The disparity maps (a) and (d) produced by a regular stereo, (b) and (e) estimated using the an attenuation coefficient that was manually estimated, and (c) and (f) obtained with parameters automatically estimated using our methodology. A significant improvement can be seen in the disparity values for the square object. Observe in images (c) and (f) how the dark region, which is erroneously indicated as being farther away, is correctly shown in gray when our underwater methodology is used.

estimated. Future research will address the two main constraints currently imposed to method by improving the model structure and by introducing an iterative procedure to infer scene depth.

\section{REFERENCES}

[1] G. Foresti, "Visual inspection of sea bottom structures by an autonomous underwater vehicle," IEEE Transactions on Systems, Man, and Cybernetics - Part B, vol. 31, no. 5, pp. 691-705, Oct. 2001.

[2] S. M. Luria and J. A. Kinney, "Underwater vision," Science, vol. 167, pp. 1454-1461, 1970.

[3] S. Negahdaripour and C. Yu, Underwater Robotic vehicles: Design and Control. TSI Press, 1995.

[4] A. Trucco and V. Murino, "Guest editors introduction : Special issue on underwater computer vision and pattern recognition," Comput. Vis. Image Underst., vol. 79, no. 1, July 2000 .

[5] M. Chyba, N. E. Leonard, and E. D. Sontag, "Optimality for underwater vehicles," in Proc. IEEE Conf. on Decision and Control, 2001, pp. 4204-4209.
[6] M. Purcell, C. von Alt, B. Allen, T. Austin, N. Forrester, R. Goldsborough, and R. Stokey, "New capabilities of the remus autonomous underwater vehicle," in Proc. MTS/IEEE Oceans'00, Providence, RI, vol. 1. IEEE, Sep. 2000, pp. $147-151$.

[7] C. Woosey and N. E. Leonard, "Moving mass control for underwater vehicles," in Proc. American Control Conference, 2002, pp. 2824-2829.

[8] Y. Kahanov and J. Royal, "Analysis of hull reamins of the dor d vessel, tantura lagoon, israel," in Int. J. Nautical Archeology, 2001, pp. 257-265.

[9] S. Bazeille, I. Quidu, L. Jaulin, and J. Malkasse, "Une mthode de pr-traitement automatique pour le dbruitage des images sous-marines," Traitement du Signal, vol. 25, no. 1-2 SP, pp. 45-54, 2008.

[10] M. Chambah, D. Semani, A. Renouf, P. Courtellemont, and A. Rizzi, "Underwater color constancy: enhancement of automatic live fish recognition," in Proc. SPIE, vol. 5293, Dec. 2003, pp. 157-168.

[11] R. Garcia, T. Nicosevici, and X. Cufi, "On the way to solve lighting problems in underwater imaging," in Proc. MTS/IEEE 
OCEANS'02, Biloxi, MS, vol. 2. IEEE, Oct. 2002, pp. 10181024.

[12] R. Eustice, O. Pizarro, H. Singh, and J. Howland, "UWIT: underwater image toolbox for optical image processing and mosaicking in MATLAB," in Proc. 2002 Intl. Symp. on Underwater Technology, 2002, pp. 141-145.

[13] Y. Y. Schechner and N. Karpel, "Clear underwater vision," in Proc. IEEE Intl.Conf. on Computer Vision and Pattern Recognition, vol. 1, 2004, pp. 536-543.

[14] M. Levoy, B. Chen, V. Vaish, M. Horowitz, I. McDowall, and M. Bolas, "Synthetic aperture confocal imaging," ACM Trans. Graph., vol. 23, no. 3, pp. 825-834, 2004.

[15] T. Treibitz and Y. Y. Schechner, "Instant 3descatter," in Proc. IEEE Intl.Conf. on Computer Vision and Pattern Recognition. Washington, DC, USA: IEEE Computer Society, 2006, pp. 1861-1868.

[16] S. G. Narasimhan and S. K. Nayar, "Vision and the atmosphere," Intl. J. Comp. Vision, vol. 48, no. 3, pp. 233-254, 2002.

[17] J. P. Queiroz Neto, R. Carceroni, W. Barros, and M. Campos, "Underwater stereo," in Proc. XVII SIBGRAPI. IEEE Computer Society, 2004, pp. 170-177.

[18] Y. Y. Schechner and N. Karpel, "Recovery of underwater visibility and structure by polarization analysis," IEEE J. of Oceanic Engineering, vol. 30, no. 3, pp. 570-587, Jul. 2005.

[19] M. Bouguer, Traite d'optique sur la gradation de la lumiere. Paris: De l'imprimerie de H.L. Guerin et L.F. Delatour, 1760.

[20] J. Zaneveld, M. Twardowski, A. Barnard, and M. Lewis, Remote Sensing of Coastal Aquatic Environments: Technologies, Techniques and Applications. Springer, 2005, ch. Introduction to Radiative Transfer, pp. 1-21.

[21] S. Narasimhan and S. Nayar, "Contrast restoration of weather degraded images," Trans. on Pat. Analysis and Machine Intell., vol. 25, no. 6, pp. 713-724, Jun. 2003.

[22] V. Kolmogorov and R. Zabih, "What energy functions can be minimized via graph cuts?" Trans. on Pat. Analysis and Machine Intell., vol. 26, no. 2, pp. 147-159, 2004.

[23] E. on Software, "Vue 6 infinite," http://www.eonsoftware.com/, 2009.

[24] D. Scharstein and R. Szeliski, "A taxonomy and evaluation of dense two-frame stereo correspondence algorithms," Intl. J. Comp. Vision, vol. 47, pp. 7-42, 2001.

[25] Point Grey Research, "Imaging products dragonfly," http://www.ptgrey.com/products/dragonfly/index.asp, 2008.

[26] J. WS and M. WR., "Aquatic vision and the modulation transfer properties of unlighted and diffusely lighted natural waters." in Vis. Res., vol. 35, 1993, pp. 1755-1763. 